\title{
Study on Clothing Structural Design and Modeling
}

\author{
Yue $\operatorname{Min}^{1}$ \\ ${ }^{1}$ Jiangxi Institute of Fashion Technology, Nanchang Jiangxi, China, 330201
}

KEYWORDS: Clothing Structural Design, Modeling, Study

\begin{abstract}
Rapid socio-economic development, people's living standards rising spirit, the pursuit of more and more obvious to clothing, this situation led directly to clothing design industry and the rapid development of derivatives. People for the pursuit of fashion design is mainly reflected in the colors, materials and workmanship, the most important is the performance of the overall styling of the garment processing, clothing people want to be able to fully reflect the taste of their own emotions and to achieve beauty perfect interpretation. This paper studies the relationship between fashion design and styling characteristics, it is desirable to combine its internal cohesion to realize the beauty of the shape of clothing, so as to design a good-quality clothing. This article briefly discusses this purpose by reference.
\end{abstract}

\section{Introduction}

Costume design work which, costume design is very important, usually divided into draping system board design and graphic design two ways, the way in which the flat plate system was more widely used in China. But whether in the draping system board or in the plane which you must use to three-dimensional modeling of the way, especially when for some relatively complex and unique creative structure, usually three-dimensional modeling application will directly play a very significant effect . Therefore, it is necessary to design requirements of the relevant personnel should exercise as much as possible the ability to design and spatial imagination, and thus its effective use in clothing structure designs, leading to the entire garment design can truly achieve the most perfect design effect.

\section{Modeling Analysis of Garment Design}

Costumes can usually be divided into inner and outer shape modeling of two types, which mainly refers to the shape of the inner garment itself has inherent style, the bag contains a specific type, structure lines, collar and dart like. The clothing is mainly refers to the outer shape of the clothing itself has contours and silhouettes, and the outer shape of the body itself is also important when designing clothing, usually require inherent in the design of the shape of the process must fully meet their exterior style features. Therefore, both the outer shape and the inner shape of the whole is always in a complementary state, which requires the design of clothing at the same time its structure, it should be possible to neglect their internal carved in the shape, while fully taking into account the two effective styling unity, in order to prevent and avoid distracting generation phenomenon.

Costume design is a unique type of visual art, usually clothes designed contour and shape will directly gives a very intuitive feeling, so in fashion design process, the design of which tend to occupy the most important position. Usually the outer garment silhouettes are divided into four categories AHXY and four different types on the basis of refined extension, in order to effectively design a variety of different costumes [1]. A garment such as binding on the shape of the line may 
extend directly out of the tent and speaker wire, etc., for the other three types of thinning will also get a similar effect different. However, if in the plane of the fabric directly reflect the three-dimensional effect, which must be to direct the removal of unnecessary components, and thus take a very malleable fabrics shaping process, generally take highway and set Tuck to fulfill.

Clothing structure during the design process usually requires strict accordance body features a variety of body styles and style design, the flexibility of the wire clips and dart pleats unified and effective coordination within the structure and the outer structure, in order to truly promote the entire costumes become more abundant. Clothing design includes specific clothing collar and sleeves effective design, because the collar on clothing belonging to a very important part, not only have the appropriate functional characteristics, but can play a fun decorative effect. The clothing collar design includes a variety of specific changes, including flat collar, collar, stand-up collar and pleated collar and other types, but also the shape of the rotator cuff has a variety of changes, to both sleeves and armhole, sleeves and collar good design can really effectively meet people's own clothing temperament and overall style.

\section{Analysis on Problems of Costume and Clothing Structural Design}

Costume designer during the design process, usually because of lack of structure of the two-dimensional plane and modeling technique of understanding, leading to its usually only designed for the plane structure, which directly ignore the three-dimensional structure of clothing design. This situation is more common, it is easy to cause some direct impact on the entire fashion design process, prompting designers to design clothing for the entire holding recognize a one-sided understanding of the final severely hampered the development of the fashion style design and processes.

Most designers to make clothing design at the same time, it is easy to overlook the direct structure of the garment design features, in this case the current design industry which is very common [2]. Even many clothing designers in the implementation of the structural design, while the design itself, the concept of lack of knowledge, lack of a clear understanding of the structural design of clothing. They typically occur frequently in fashion design should not exist among some of the phenomenon, which led directly to clothing design which produce a series of unreasonable conditions, which seriously hampered the overall development of clothing structure design. Therefore, in the costume design process requires the designer must be fully recognized for its function, this is certainly an effective way to really solve the problem.

Used in clothing design work among the entire curve design effect is extremely important, but it also directly into the main difficulties of the designers design work. Contains specific curve design curve and the outer edge of the inner garment of two curves design approach, like the design of the collar and cuffs of clothing belonging to the internal structure design, designers in the actual structure of the clothing designs, usually encounter such problems, but due to a serious shortage of appropriate strategies and programs practicality, it is difficult to really get an effective solution, and ultimately led directly to the designer in the design of clothing structure while it is difficult to grasp the real problems and difficulties of curve designs.

\section{Strategies to Achieve Clothing Structural Design and Modeling Fashion}

Clothing structural design is the garment flat structure stuff directly into the three-dimensional modeling of the space, which requires the relevant design must be in costume to carry out the structural design process, the need to strengthen the designers themselves perspective thinking 
ability, way of thinking and on this basis to rationalize the application of scientific perspective to effectively solve the design and styling which difficult problems. Because the clothing itself is not only an important component of a planar structure which also belongs to the entity exists among the three-dimensional space, but the actual modeling costumes talking about here is the space itself among. Therefore, to carry out the process designer clothing structure design, it should be considered from many angles, to assess the overall analysis of the shape of clothing, emphasis on three-dimensional thinking of good applications, take advantage of this expansion of the clothing and other details of the design and structure depth study of aspects.

Three-dimensional structure composed is well known designers who constitute the space, and the main object of study or in three-dimensional space formed structural aspects of the law of costumes, which itself played out in the structural design of clothing among an extremely important role. In addition, three-dimensional constitution itself mean for designers and creative thinking perspective and three-dimensional garment design effect is of great significance. The costume designer to design the structure, we need to start from the simplest shapes directly, in order to develop a fully three-dimensional modeling capabilities of its structure, such as in the garment structure among the drape, waves and folds like. Also directly through a variety of three-dimensional form of equipment and tools to enhance the look and feel of the effect of three-dimensional structure, so has obvious significance for the formation of designers thinking in terms of spatial modeling [3]. In addition, the designer after the completion of the appropriate exercise really work, a sense of space its design will greatly increase, eventually will be better to deal with some of the complicated costume design requirements.

Costume design to achieve specific needs from the standpoint of human form, and thus to shape the modeling process, and for the beauty of the clothing, its spatial modeling results for the clothing aesthetic shape played a very important role. When it is on the costume design process, a simple planar system board is often difficult to achieve its desired design effect. This requires a three-dimensional design composed primarily requires the designer must have the appropriate spatial modeling capabilities, and in some practical fashion design which should be strictly focusing on costume design aesthetic itself, under the premise of ensuring compliance with the requirements of clothing styles, using a variety of effective ways to really promote the reunification of their costumes and effects.

\section{Conclusion}

To sum up, costumes and structural design two complement each other, in which there are some obvious problems, but in order to truly realize the clothing design and modeling to create the designer need to strengthen the three-dimensional thinking, strengthen the construction of three-dimensional modeling clothing effective expansion and greatly enhance the designer space modeling capabilities.

\section{References}

[1] Jin Shuang. Mu Xuemei. Garment Design three-dimensional modeling Ability [J]. Arts Education, 2013,12: 186.

[2] Liang Jun. package width original style and structure costume design Teaching [J]. Art hundreds, 2012, S1:.. 398-399.

[3] Lai Yiping. role of film and television costume design in architecture [J]. Movie Review, 2015,09: 104-105. 